\title{
FEPACS: A computational tool for linear structural analysis
}

\author{
B P NAGANARAYANA, G PRATHAP and B R SOMASHEKAR \\ Structures Division, National Aerospace Laboratories, Bangalore 560017 , \\ India \\ email: [naga,gp,brs]@csnal.ren.nic.in
}

\begin{abstract}
FEPACS (Finite Element Package for linear static, dynamic and instability Analysis of Composite Structures under hygro-thermo-mechanical loads) incorporates a complete library of consistent and correct 1-, 2- and 3dimensional linear and quadratic general purpose finite elements. In this paper, we shall discuss the finite element technology that has gone into the package as well as its present modelling and solution capabilities. We shall also discuss briefly recent developments toward enhancing the package: Robust composite elements based on a $C^{0}$-continuous higher order transverse deformation plate/beam theory, and nonlinear element technology and solution strategies. Finally, we shall also briefly touch upon several satellite application modules that are in different stages of planning/development to aid FEPACS: damage assessment/prediction, expert-like advisors for solid modelling and finite element modelling/analysis, pre-/post-processing for FEPACS applications, structural optimisation and related finite element algorithms, and finally, a frontal solution module for FEPACS to enhance its feasibility for vectorisation/parallelisation.
\end{abstract}

Keywords. Analysis of composite structures; hygro-thermo-mechanical loads; linear structural analysis, finite element package.

\section{Prelude}

Today, due to very high demands on functional excellence and technological perfection, availability of advanced material and design technology, the advent of advanced mathematical and computational tools and computer technology, and finally due to stringent safety and economical constraints, precise and accurate prediction of structural behaviour and strength under complex and adverse environment is called for, particularly with reference to aerospace and automobile applications. The finite element method (FEM) appears to be the only computational tool that can be used successfully for high precision structural analysis meeting current demands as well as expectations in the near future.

Due to its versatility and simplicity in applications, finite element analysis (FEA) has attracted researchers from many different engineering fields, e.g. aerospace, civil, mechanical, automobile, electrical and so on, particularly with reference to structural applications, 
as well as in other branches of science and mathematics in the past four decades of its history. It started off as a technological art with matrix methods in the late 50's and since then has progressed through the intense research efforts of scientists all over the world. Today, the finite element method may have more than 400 books, 450 conference proceedings, 70 journals, and 65000 journal papers written about it (projected from Mackerle 1995). Currently, 3500-4000 papers are being published every year on the finite element method. Because of the enormous research and developmental attention it has received, finite element analysis has now grown to an acceptably matured status from the points of view of both technology as well as science.

It is due to the scientific and technological maturity which finite element analysis has attained today that one could take up the tasks of reliable design/analysis in many complex real-life structural applications like: static/stability/dynamic analysis of structures under real-life environment, geometric/material nonlinear structural behaviour, fatigue and fracture/damage mechanics, hygro-thermal applications, design/analysis of advanced mate$\mathrm{rial} / \mathrm{structural}$ constitution, fluid-structural interaction (e.g. sloshing, aeroelasticity, flutter etc.), acoustic-structural interaction, structural optimisation, adaptive structures and design, material health monitoring, structural crash-worthiness, contact (friction) problems, and finally, possible interaction between any of the above mechanisms/applications. Several fields have emerged which enhance the utility of FEM as well, expert systems, adaptive mesh control, CAD/CAM Graphical User Interface etc.

Due to the wide range and generality of applications, general purpose packages play a very important role in structural design and analysis as well as in many other applications. Several in-house and commercial finite element packages have been developed all over the world for specific categories of engineering and scientific applications. According to Mackerle (1995), there are now nearly 1500 established in-house finite element packages out of which more than 300 are general purpose in nature. A few of the successful commercial finite element packages are MSC/NASTRAN, ABAQUS, NISA, ANSYS and ALGOR. Efforts to develop useful general-purpose finite element packages are going on in India as well, e.g. FEAST (Vikram Sarabhai Scientific Centre, Thiruvananthapuram), FINESSE (Aeronautical Development Agency, Bangalore), GSAFE (Godrej, India Ltd.), FEPACS (National Aerospace Laboratories (NAL), Bangalore) with varying degrees of success.

Most of the finite element packages all over the world use finite element technology based on $C^{0}$-continuous field description since such elements require only engineering degrees of freedom specified in a unique Cartesian coordinate system. This makes finite element modelling simple, efficient and general, particularly with reference to large-scale general structures. However, such $C^{0}$-continuous elements can suffer from very severe problems like locking and stress oscillations under certain operating conditions. To overcome these problems, most of the finite element formulations available in the literature use many ad-hoc methodologies (e.g. reduced integration) which often lack sound scientific foundation and offer only partial success. Accordingly, the functional adequacy of the element technology used in such packages is not completely fool-proof and may fail in one or the other circumstance.

In the late 70 's and early 80 's, the scientific value of the available methodologies and explanations regarding the perennial problems like locking were critically re-examined at NAL, Bangalore. In the process, a complete, consistent and correct methodology was 
evolved for formulating robust finite elements that are free of all the errors under any circumstances. A library of general purpose linear and quadratic finite elements are developed for 1-, 2- and 3-dimensional structural modelling/analysis. FEPACS uses such a scientifically proven element technology and incorporates a complete library of state-of-the-art robust elements. We review this work here.

\section{Introduction}

Understandably, displacement type finite elements have been the standard for developing the element library of any general purpose package since such formulations are simple to code and economical to use. In addition, the displacement type elements can be made robust (simple, but efficient) even in their distorted configurations. Also, in a general purpose finite element software, $C^{0}$-continuous element formulations are sought since such formulations can satisfy the required continuity conditions exactly and allow one to use only the engineering degrees of freedom to model a problem. In the case of flexural elements (e.g. beams, plates and shells), they permit modelling of transverse deformation (e.g. transverse shear) which becomes important in case of laminated and/or thick structures. The first $C^{0}$-continuous finite element (flexural) formulation was developed in the late 60's - the 8-noded quadratic degenerated shell element (Ahmed et al 1970).

However, soon after they were discovered, the $C^{0}$-continuous flexural elements were found to be crippled with dramatic failures such as shear locking and stress oscillations even when the conventional continuity and completeness requirements were satisfied (Doherty et al 1969; Pawsey \& Clough 1971; Zienkiewicz et al 1971). Later, similar situations were recognised - membrane locking in curved elements (Stolarski \& Belytchko 1981), parasitic shear (Cook 1975), incompressibility locking (Fried 1974), nonlinear locking (Naganarayana \& Prathap 1996) etc. Many ad-hoc techniques are offered in the literature to alleviate these different types of locking - reduced/selective integration (Pawsey \& Clough 1971; Zienkiewicz et al 1971), assumed strain methods (MacNeal 1982; Prathap 1993), addition of incompatible (bubble) modes (Wilson et al 1973), quasi-conforming techniques (Tang et al 1984), mixed/hybrid formulations (Pian 1971), to name a few - with varied success. Often these methods lacked a scientific explanation either for their success or for their failure. Though some attempts were made to explain locking - singularity of shear stiffness (Zienkiewicz 1977), constraint counting and rank of the shear stiffness (Cook et al 1981; Hughes 1987) - they generally tried to locate the symptoms of the problem rather than the cause (Prathap 1986). They lacked a scientific basis and could not explain the locking phenomena in a unified sense. Finally, they never offered any methodology for eliminating the locking errors, or, in other words, they could not prove why certain ad-hoc techniques could eliminate locking under certain circumstances.

In the early 80's (Prathap \& Bhashyam 1982), a scientifically valid paradigm was introduced to explain the existence of locking in a Timoshenko beam element and the success of the reduced integration technique. Over the next fifteen years, this paradigm matured as a scientific principle - field consistency - in the finite element method offering explanations for the existence of locking, delayed convergence and stress oscillations in the so-called 
class of constrained media elasticity (Prathap 1986) and a library of field-consistent linear elements - elements with linear field interpolations - was developed (Ramesh Babu 1985; Ramesh Babu et al 1985).

Later, the field-consistency paradigm was implemented in several quadratic finite elements. New consistency paradigms were offered to explain the difficulties that arose here and to provide methods to eliminate these errors - consistent mapping (Prathap \& Naganarayana 1992), edge consistency (Prathap \& Somashekar 1988), stress/initial-strain field consistency (Prathap \& Naganarayana 1990a), warping correction (Naganarayana \& Prathap 1989a), and variational correctness (Prathap 1988). These principles are critically examined and implemented in several general purpose quadratic displacement type 1-, 2and 3-dimensional elements (Naganarayana 1991). A complete library of robust, consistent and correct linear and quadratic linear elements is now available (Prathap et al 1994a). An extensive treatment of the different paradigms, apart from the classical continuity and completeness requirements, was given recently by Prathap $(1993,1994)$.

This library of robust linear and quadratic 1-, 2- and 3-dimensienal elements, was proposed to be implemented in a general purpose finite element platform using the solution capabilities and data management of SAP-IV (SAP - structural analys is program) which was available in source in public domain (Prathap et al 1989) for modelling and analysing advanced anisotropic and laminated composite structures effectively. Initially, the basic laminated composite beam and shell elements were implemented in the SAP-IV infrastructure on a UNIVAC system The complete element library was subsequently implemented in the UNIX operating system on PC386/486 platforms and on workstations. The first version of the package - FEPACS: Finite Element Package for Analysis of Composite Structures - was released in 1991 for linear static and dynamic structural analysis on a PC386/486 platform in a UNIX environment (Prathap \& Naganarayana 1991). Later, the eigenvalue solution capability was enhanced to accommodate the consistent mass description and then extended for analysis of structural instability (Naganarayana et al 1993). The current version, FEPACS-2.0 (Prathap et al 1994b), has more than 15,000 (executable) lines of in-house developed code out of about 20,000 lines. A diagrammatic description of the package is given in figure 1.

Currently, several satellite modules tailored around FEPACS-2.0 are initiated at the National Aerospace Laboratories, Bangalore - expert advised finite element modelling and adaptive mesh refinement, pre- and post-processors, damage/failure mechanics, nonlinear structural mechanics and automated post-buckling solution capability, robust higher order shear flexible finite elements, hygro-thermal effects on structural behaviour, structural optimisation, and frontal solution modules for linear structural analysis.

In this paper, the state-of-the-art finite eiement technology and the finite element library in FEPACS-2.0, and the satellite structural modelling/analysis modules that have been currently developed/planned for FEPACS applications are briefly described.

\section{Finite element technology - the C-concepts}

In this section, we shall discuss the state-of-art finite element technology of FEPACS and its scientific foundations. 


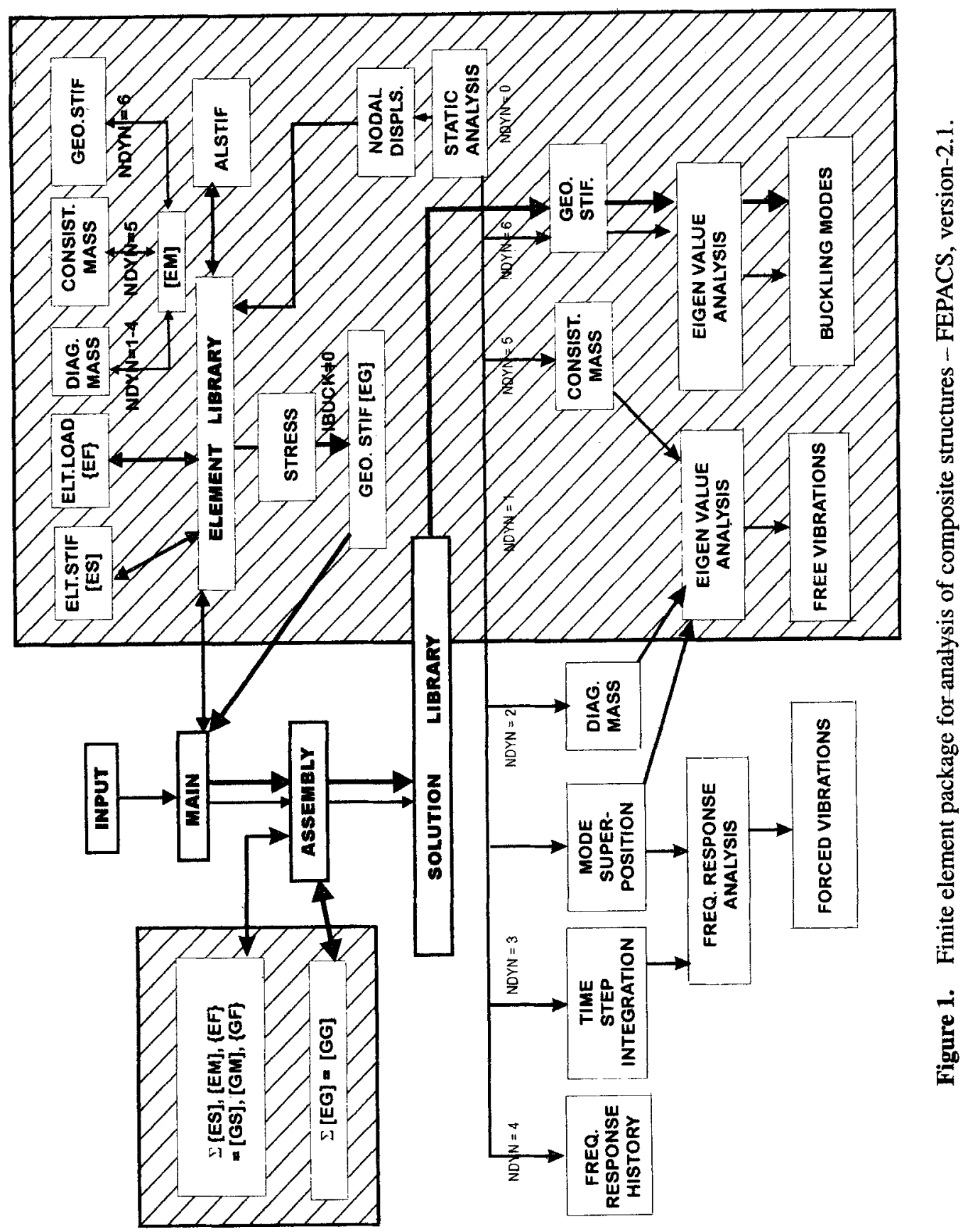


In the finite element method, we discretise the continuum/structure into a number of finite sized divisions called elements; capture the basic equations of solid/structural mechanics. The equations include strain-displacement relations such that compatibility conditions are satisfied over the element domain, the stress-strain (constitutive) relations - and the element loads to form total potential energy (complementary energy in case of stress type formulations) of each element; assemble the element total potential energy such that the compatibility conditions are satisfied across the element boundaries and the boundary conditions on the structural boundary are satisfied, apply minimum total potential energy principle (minimum complementary energy principle in case of stress type formulations) to obtain global equilibrium equations as a set of simultaneous algebraic equations (one associated with each unspecified degree of freedom in the finite element model in terms structural stiffness, nodal degrees of freedom and nodal forces) which can be solved using any established numerical method. Out of several approaches that are proposed in the literature, the displacement type approach is used now for most general purpose structural applications.

It is usually believed that finite element analysis produces discretized displacement fields which are approximations of the actual solution and the strains and stresses are then derived as the derivatives of the displacement field in each element - displacement correspondence paradigm (e.g. Barlow 1976, MacNeal 1994). Hence, it is believed that the displacements are sampled to the most accurate order in a finite element and that strains and stresses are always computed to one order less than that of displacement recovery. However, recent studies indicate that it is the strain field that is always directly sampled to form strain energy in energy-based methods. Hence, it is argued that, in the finite element analysis, a best-fit solution is always sought for the strain and stress fields within an element and the displacement field is computed in an integral sense from these sampled values of the strains and stresses such that the minimum total potential energy principle is satisfied for the assembled finite element model - this can be called the stress correspondence paradigm (Prathap 1996).

Once the basis of finite element method (for structural analysis, in particular) is understood in terms of the stress correspondence paradigm, we still face the questions: How accurate can the finite element method be? What are the prerequisites for assuring the correct convergence rate for a given finite element model? To answer these questions, we need to examine how the element strain-displacement relations are formed since the rest of the method is well understood. Conventionally, it is argued that, the mathematically expected convergence rate could be sought in a finite element model by satisfying the so-called continuity and completeness conditions, which can be broadly described as follows.

The Continuity condition requires that the interpolation functions chosen for each field variable must be such that the corresponding field and its successive derivatives upto $(n-1)$ th order should be continuous over the element and the same has to be ensured across the element boundary as well where $n$th order derivative of the field is required to define the structural strain energy. Accordingly, the formulation is referred to as $C^{n-1}$ continuous.

The Completeness condition requires that the field interpolations chosen should be such that rigid body motion should not produce strains and constant strain states of the element 
Table 1. Types of errors, their sources in finite element analysis and associated paradigms.

\begin{tabular}{|c|c|c|}
\hline Source & Symptoms & Paradigm/concepts \\
\hline $\begin{array}{l}\text { Errors of first kind } \\
\text { Finite element } \\
\text { discretisation }\end{array}$ & Discretisation errors & $\begin{array}{l}\text { Continuity and } \\
\text { completeness }\end{array}$ \\
\hline $\begin{array}{l}\text { Errors of second kind } \\
\text { Constrained } \\
\text { media elasticity }\end{array}$ & $\begin{array}{l}\text { Locking, } \\
\text { delayed convergence, } \\
\text { stress oscillations }\end{array}$ & $\begin{array}{l}\text { Constrained-field } \\
\text { consistency }\end{array}$ \\
\hline $\begin{array}{l}\text { Element distortion } \\
\text { (nonuniform mapping) }\end{array}$ & $\begin{array}{l}\text { Locking, } \\
\text { delayed convergence, } \\
\text { stress oscillations }\end{array}$ & $\begin{array}{l}\text { Edge consistency and } \\
\text { consistent mapping }\end{array}$ \\
\hline $\begin{array}{l}\text { Varying material/ } \\
\text { sectional modulii }\end{array}$ & $\begin{array}{l}\text { Stress } \\
\text { oscillations }\end{array}$ & $\begin{array}{l}\text { Unconstrained-field } \\
\text { consistency }\end{array}$ \\
\hline $\begin{array}{l}\text { Initial-strain/ } \\
\text { initial-stress } \\
\text { representation }\end{array}$ & $\begin{array}{l}\text { Stress } \\
\text { oscillations }\end{array}$ & $\begin{array}{l}\text { Unconstrained-field } \\
\text { consistency }\end{array}$ \\
\hline $\begin{array}{l}\text { Reconstitution of } \\
\text { strain/stress } \\
\text { fields }\end{array}$ & $\begin{array}{l}\text { Poorer convergence, } \\
\text { spurious load mech- } \\
\text { anisms and stress } \\
\text { oscillations }\end{array}$ & $\begin{array}{l}\text { Variational } \\
\text { Correctness }\end{array}$ \\
\hline $\begin{array}{l}\text { Modelling warped } \\
\text { surface with plane } \\
\text { elements }\end{array}$ & $\begin{array}{l}\text { Erroneous stresses } \\
\text { and displacements }\end{array}$ & $\begin{array}{l}\text { Warping correction } \\
\text { (minimum virtual } \\
\text { work principle) }\end{array}$ \\
\hline
\end{tabular}

should be represented and that no intermediate polynomial terms should be dropped while interpolating the displacement fields.

It was conventional wisdom that the finite element formulations that satisfy the above continuity and completeness conditions shall converge in a variationally correct rate. However, subsequently it was found that the finite elements suffer from many problems such as locking, delayed convergence, and stress oscillations when applied a large class of problems - constrained media elasticity where at least one or more components of the strain tensor are constrained at a physical limit and many other classes of problems (table 1). These problems make element formulations virtually unacceptable for general structural applications. Several ad hoc techniques such as reduced integration, mixed/hybrid methods, addition of incompatibility modes etc. were normally associated with stress sampling at the so-called optimal/Barlow points. All these techniques offered only selective/partial success from general purpose point of view and lacked a scientifically sound explanation either for their success or for their failure (if any). Basically, the solutions offered to the 
basic questions - How confident are we with finite element analysis? and How reliable are the finite element solutions? - were not completely satisfying.

In the early 80 's, this class of problems was approached with a new scientific understanding (Prathap \& Bhashyam 1982) and over the past one and half decades a complete consistent and correct scientific basis is established for formulating any general purpose robust finite element for structural applications (e.g. Ramesh Babu (1985), Prathap (1986, 1993,1994), Naganarayana (1991), Prathap et al (1994a)). In this process, several classes of structural problems which suffer from errors like locking, delayed convergence and stress oscillations in the general purpose finite element formulations were identified; appropriate consistency principles were evolved to explain the existence of such errors, their root causes and possible method of eliminating the same in a variationally correct manner (table 1). Several techniques such as reduced integration, least square field-reconstitution, Legendre polynomial expansion can now be reinterpreted with the new scientific rigour for applications in finite element formulations. Here, we shall briefly recapitulate the different aspects of the new understanding - consistency and correctness paradigms.

Constrained-field consistency: The displacement fields involved in computing the constrained strain energy components should be interpolated such that all the physical constraints on the corresponding strain energy component are fully represented without leading to any spurious constraints in the penalty limits. In other words, the terms in a constrained strain field that have partial contribution from the constituent displacement fields, leading to the spurious constraints in the penalty limits for the corresponding strain energy components, should be eliminated from the formulation for assuring the expected rate of convergence from a finite element model of the structural problems belonging to the class of constrained media elasticity.

Consistent Mapping: In any parametric formulation, mapping of the strain and stress fields from the natural system (the system in which the element configuration is always undistorted and the displacement fields are interpolated) to the working system (the system in which the problem is defined and the solution is sought for) should retain all the true discretised constraints without introducing any additional spurious ones.

Edge-consistency: The tangential strain components which are continuous across the element boundary in the undistorted natural coordinate system should remain continuous even after the necessary transformations; and the tangential strain components should be built from their corresponding tangential displacement components only.

Unconstrained-field consistency: The terms in the strain and/or stress fields that do not participate in the strain energy computations (and hence in the displacement recovery) should not be retained while recovering the corresponding strains and/or stresses in a displacement type formulation.

All the above consistency paradigms suggest some form of field-reconstitution (either strain or stress fields) to eliminate the associated errors such as locking and stress 
oscillations. There are many ad hoc schemes (e.g. reduced integration) to perform the same. The field-reconstitution, however, should be performed in accordance with the following variational basis.

Variational correctness: The reconstituted field should be orthogonal to the error field being introduced in the reconstituted field with reference to the original field.

The above paradigms - Correspondence, Continuity, Completeness, Consistency and Correctness, collectively called the C-concepts - form a complete scientific basis for robust finite element formulations (figure 2).

\section{Finite element library}

The Structural Analysis Program (SAP) is one of the earliest general purpose finite element programs used for structural analysis (Wilson 1970). This was later improved and released as SAP-IV for linear static and dynamic analysis of 3-dimensional structures (Bathe et al 1974). As it has been released with its source code in the public domain, it has served as the spring board for many other finite element packages with various improvements. It is well known that SAP-IV has reasonably efficient solution capabilities and data handling procedures. However, its main weakness is its obsolete finite element technology and the limited element library (only linear elements that can model only isotropic structures). The idea of developing a general purpose finite element package with the strength of the state-of-the-art finite element technology available in the National Aerospace Laboratories with an emphasis on application to the laminated composite structures under hygro-thermo-mechanical loads was conceived in the late 80's. FEPACS: Finite Element Package for Analysis of laminated Composite Structures under hygro-thermo-mechanical loads (Prathap et al 1989) was initiated with this in mind. Initially, the package was built around the data handling and program organisation of SAP-IV. Over the years, the element library was replaced with a complete library of the consistent and correct 1-, 2- and 3-dimensional, linear and quadratic displacement type finite elements. In this section, we shall briefly describe the salient features of each element in the FEPACS library (figure 3) for linear analysis of composite structures.

\subsection{The boundary element}

SPRING - A 2-noded spring/boundary element: This is a simple spring element-defined by two nodes (one being a specified structural node and the other to fix the spring direction) in space. The spring stiffness can be prescribed along any six engineering degrees of freedom so that any general translational and/or torsional springs can be modelled with this element. Apart from the spring stiffness, it takes specified displacement along the element axis and specified rotation about the element axis. It can also be effectively used as a general boundary element allowing initial displacements (boundary conditions) on the structural node to be imposed. By using a proper combination of displacement and stiffness conditions, it can be used to emulate the multi-point constraints in a structural model, to model elastic foundations, etc. Finally, if the plate/shell element 


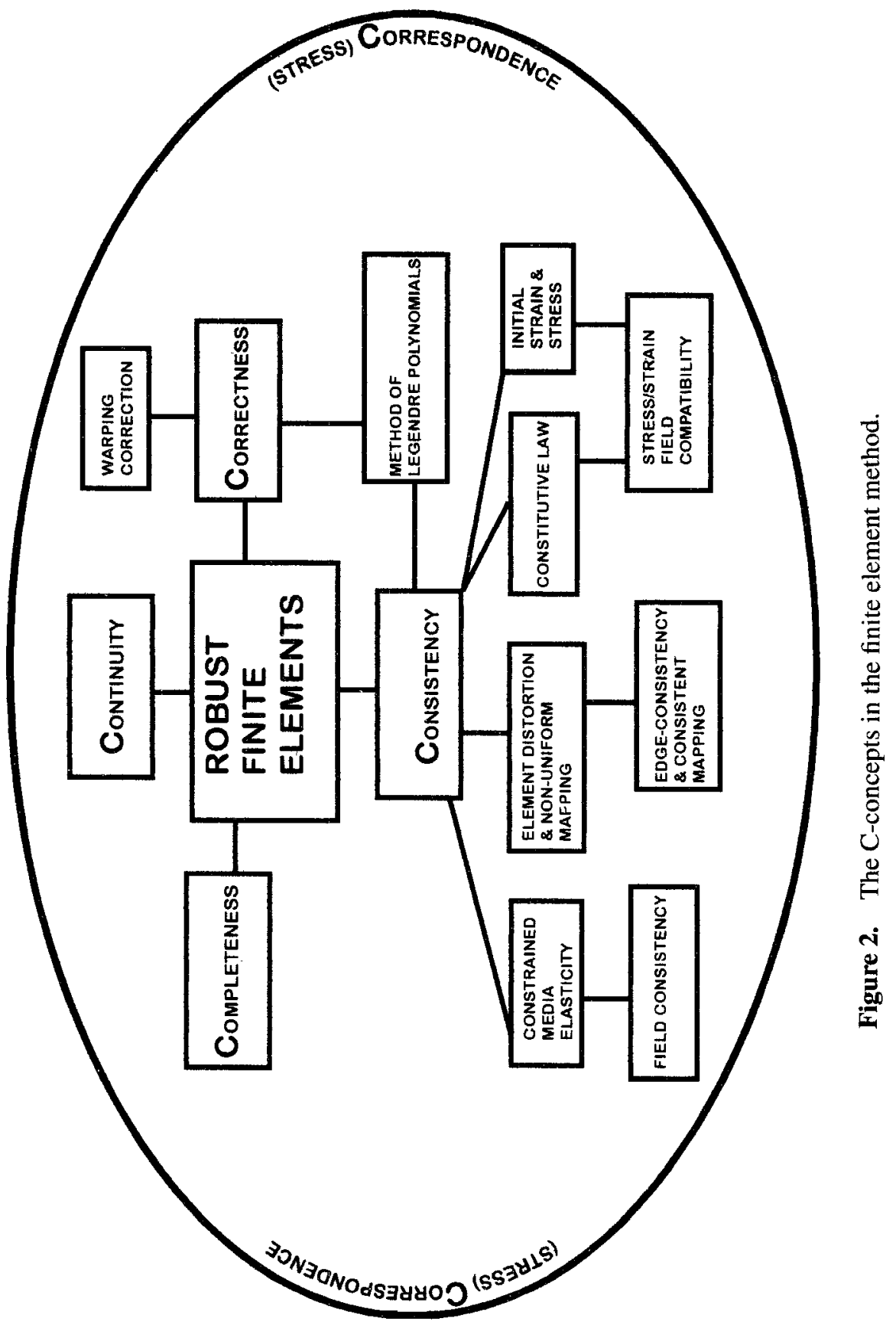



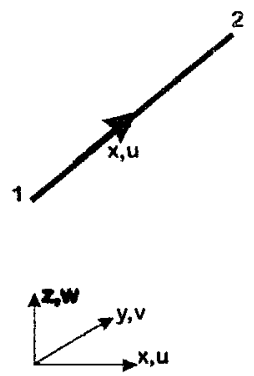

TRUSS
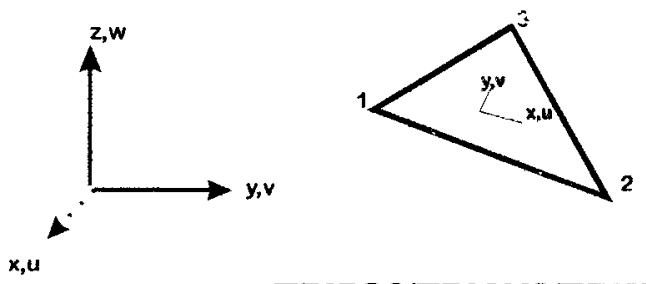

TRIPS3/TRIAX3/TRIM3

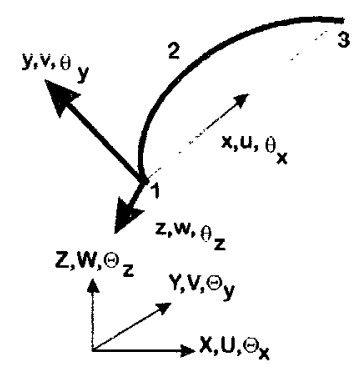

BEAM3
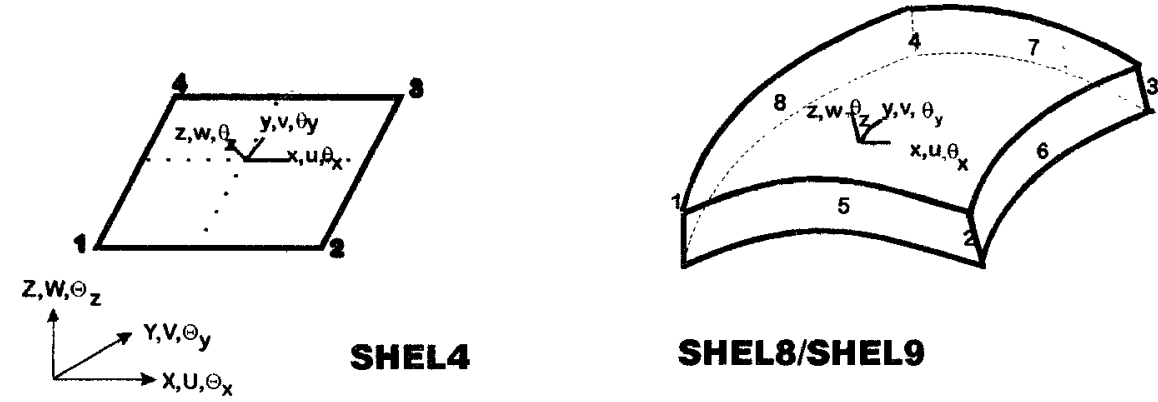

SHEL8/SHEL9

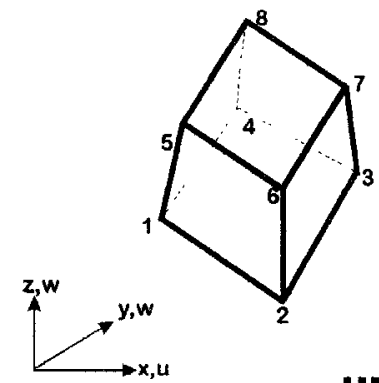

HEXA8
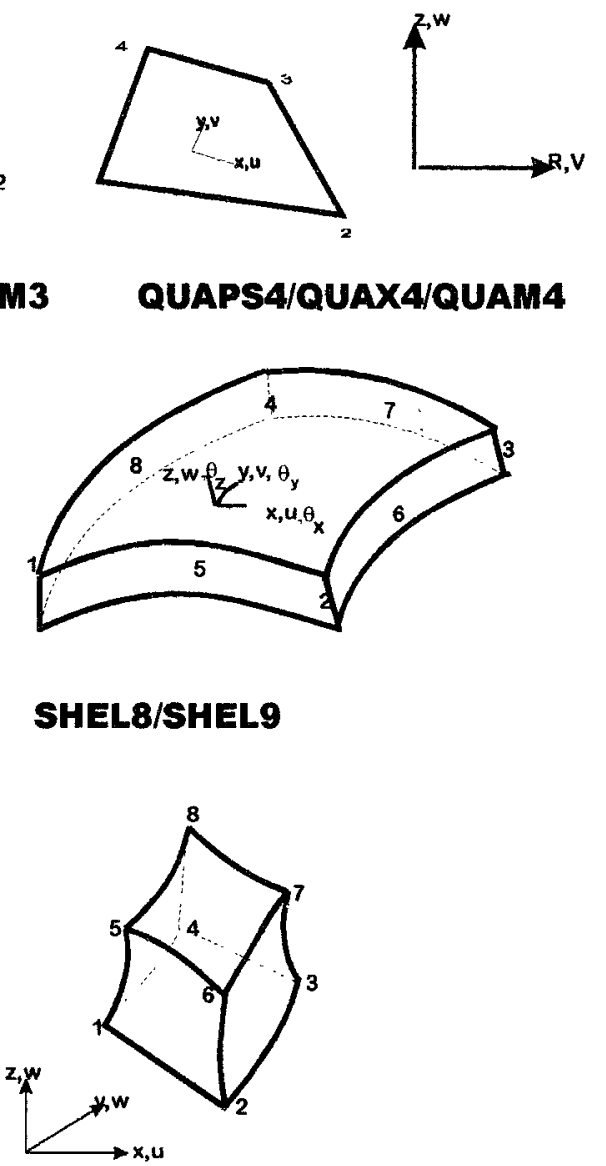

HEXA20/HEXA27

Figure 3. FEPACS-2.1: The element library. 
formulation cannot support the rotation about the local normal, the boundary element can be used to suppress this degree of freedom locally at the specified node in a general structure.

\subsection{The truss/bar element}

TRUSS - A 2-noded truss/bar element: A spatial (3-dimensional) truss element is incorporated in FEPACS. This element is based on 1-dimensional elasticity assumptions - that the structure can take loads and deform only in its axial direction - in the element coordinate system. This element does not involve any inconsistency problems in its original form and hence the conventional formulation suffices the general purpose requirement. This element can support both mechanical and thermal loads.

\subsection{The beam elements}

The 3-dimensional elasticity relations are reduced to one-dimensional relations for a beam by using the one-dimensional nature of its geometry -2-dimensions are very small when compared to the third - using different levels of approximation (theories) such as the elementary theory (Euler-Bernoulli); first-order shear deformable theory (Timoshenko); and several higher order transverse deformable theories (e.g. Lo-Christensen-Wu). In this section we shall briefly describe a family of beam elements based on the elementary and first order shear deformable theories.

All the beam elements can be used to model any general laminated composite and anisotropic beam of any geometry in 3-dimensional space. The 3-dimensional orthotropic and anisotropic constitutive relations are statically condensed to its equivalent onedimensional form. The elements can model any 3-dimensional straight/curved beam/frame structures. The elements can be loaded along all the six engineering degrees of freedom. They can also be effectively used as the stiffener elements with the plate, shell, and solid elements. The eccentricity of the stiffener can either be modelled by defining the beam nodes as slave nodes or by processing the stiffener offset explicitly. Finally, the elements can also model uniform, tapered, and stepped beams of any cross-section and curvature and are always free of all errors of locking and stress oscillations.

EBEAM2 - A 2-noded Euler-Bernoulli beam element: This element is based on the classical theory of beam fiexure (Euler-Bernoulli): the plane normal to the neutral axis remains plane and normal to the neutral axis even after deformation. Thus, the element involves only four independent fields - axial extension, axial twist, transverse deflections along two orthogonal directions in the cross-sectional plane. The transverse deflections require $C^{1}$-continuity to be satisfied while the other degrees of freedom should satisfy $\mathrm{C}^{0}$-continuity. The one-dimensional cubic Hermitian polynomials are used for interpolating the transverse deflection fields and the linear Lagrangian polynomials are used for interpolating the other degrees of freedom and the element geometry. This element is formulated in the Cartesian coordinate system to be used as a straight thin beam element. A variation has been provided in the curvilinear systems as well so that it can also be used 
as a curved thin beam element. This element, in its original form, suffers from membrane locking when used as a curved beam. However, the constrained membrane strain field is made field-consistent using the method of Legendre polynomial expansion making the element free of locking and stress oscillations.

TBEAM2-A 2-noded Timoshenko beam element: A 2-noded beam element based on the first-order transverse shear flexible theory of beam flexure that is compatible with the shell elements of FEPACS (Timoshenko): the plane normal to the neutral axis remains plane but need not be normal to the neutral axis after deformation. Thus, the element involves all the six engineering degrees of freedom - axial extension, axial twist, transverse deflections along two orthogonal directions in the cross-sectional plane and sectional rotations about the two transverse directions. All the degrees of freedom need to satisfy only $C^{0}$-continuity. The linear Lagrangian polynomials are used for interpolating all the degrees of freedom and the element geometry. This element is formulated in the Cartesian coordinate system to be used as a straight thick/thin beam element. This element, in its original form, suffers from shear locking as the beam becomes thin. However, the constrained transverse shear strain fields are made field-consistent using the method of Legendre polynomial expansion making the element free of locking and stress oscillations.

TBEAM3 - A 3-noded curved Timoshenko beam element: A 3-noded Cartesian curved beam element based on the Timoshenko theory of beam flexure that is compatible with the quadratic shell elements of FEPACS is included in the FEPACS library. The element involves all the six engineering degrees of freedom and all the degrees of freedom need to satisfy only $C^{0}$-continuity. The quadratic Lagrangian polynomials are used for interpolating all the degrees of freedom and the element geometry. This element is formulated in the Cartesian coordinate system to be used as a straight/curved thick/thin beam element. This element, in its original form, suffers from shear locking, membrane locking, delayed convergence and complex stress oscillations when the beam becomes thin and/or used to model curved beams under the conditions of inextensible flexure (Naganarayana \& Prathap 1990; Prathap \& Naganarayana 1990). To capture the constrained-field inconsistencies correctly, the element is modelled using four coordinate systems: Global Cartesian to model the structure and for displacement recovery; Element plane Cartesian for interpolating the degrees of freedom in terms of their respective nodal values; running Cartesian for capturing the strain and stress variation; and the natural curvilinear system for defining the field interpolations. The constrained strain fields (membrane and transverse shear) are made field-consistent using the method of Legendre polynomial expansion in the element plane Cartesian system making the element free of locking and stress oscillations in its general form. The strain fields are mapped from one system to another in a consistent manner so that the element behaves accurately even when the mid-node does not lie at the mid-point. The stress resultant fields are reconstituted such that they do not have any term that does not participate in the displacement recovery so that the element is free of stress oscillations even when its sectional modulii vary, e.g. tapered beams. 


\subsection{Elements for 2-dimensional elasticity}

A class of linear triangular and quadrilateral elements based on the 2-dimensional elasticity equations is included in FEPACS. All the elements are formulated in an isoparametric sense. The conventional isoparametric formulations suffer from locking (parasitic shear) and stress oscillations (Prathap 1985). Though a straightforward application of the consistency principles can alleviate these errors from the elements, a new error, Poisson's ratio stiffening, is introduced due to lack of terms that can represent flexural deformations of the elements. The linear interpolation functions have been augmented by the quadratic incompatible (the so-called bubble) functions (Wilson et al 1973) and then the consistency paradigm is applied to alleviate the problems of locking (parasitic shear), Poisson's stiffening effect, and the associated stress oscillations (Prathap 1993) from the elements. All the elements can take both mechanical and hygro-thermal loads. They can be used to model any temperature-dependent orthotropic medium.

TRIPS3, QUAPS4 - Plane-stress/plane-strain elements: These elements are based on the constitutive relationships that are derived from the theories of plane stress and plane strain respectively. They are always defined in the $y z$-plane, the $x$-axis representing the thickness or axial direction. Accordingly, they can be effectively used to model any orthotropic planestress or plane-strain problems under thermo-mechanical loads.

TRIAX3, QUAX4 - Axisymmetric solid elements: These elements are formulated based on the axisymmetric solid mechanics. The elements are defined in the $r \theta$-or $y z$-plane, the $x$-axis representing the axis of revolution. These elements can be used to model any orthotropic axisymmetric structures under axisymmetric thermo-mechanical loads.

TRIM3, QUAM4-Membrane elements: The plane-stress elements mentioned above are now defined in 3-dimensional Cartesian space so that we can model the 3-dimensional orthotropic membrane problems under thermo-mechanical loads effectively. The element is first designed in a local Cartesian plane capturing its plane-stress behaviour and the element matrices are then mapped onto the 3-dimensional Cartesian space using the regular transformations.

\subsection{Plate/shell elements}

FEPACS element library contains robust linear and quadratic plate/shell elements based on the first order shear deformable theory (Reissner-Mindlin) to enable one to model any kind of laminated composite plate or shell structures subjected to thermo-mechanical loads.

SHELA - A 4-noded plane shell element: SHEL4 is a 4-noded quadrilateral plane-shell element that can be used to model thick and thin plates/shells with equal accuracy. The element is formulated in a local mean plane defined by the mid-points of the four edges and transformed into the global Cartesian system. The original elements suffer from shear 
locking and the associated stress oscillations. To alleviate these problems the transverse shear strain fields are made consistent. The transverse shear component in the plane tangential to the element mid-surface is also explicitly matched from element to element edge-consistent formulation - such that the element performs well even when collapsed to a triangle. The effects of element warping (i.e. when the element nodes do not lie in a single plane) is captured by applying a warping correction on the element matrices based on the virtual work principle (Naganarayana \& Prathap 1989a).

SSHL8/DSHL8 - 8-noded degenerated shell elements: SSHL8 is a 8-noded element using the quadratic serendipity functions for interpolating the field-variables as well as its geometry in element plane Cartesian system. The element deformation is captured in a running local Cartesian system. The 3-dimensional elasticity equations are degenerated to 2-dimensional equations using the assumptions of the Reissner-Mindlin theory. The element is formulated in two different versions: the single-surface and the double-surface formulations. The thickness dimension of the 3-dimensional elasticity is modelled using two sets of geometric nodes on the bottom and top surfaces of the element in the double-surface formulation. This element is more efficient for modelling problems involving adjacent elements that do not share common normals at the joining nodes. On the other hand, in the single-surface formulation, the thickness dimension in the 3-dimensional elasticity equations is modelled using the normal to the element midsurface at the element nodes. Such formulations need only one set of mid-surface nodes and are convenient to use in general. The single-surface formulation is particularly efficient in modelling problems involving adjacent elements that share a common normal to their mid-surfaces.

These elements, in their conventional form, suffer from transverse shear locking, membrane locking, parasitic shear and associated stress oscillations. A special technique called line consistency (Naganarayana \& Prathap 1989b) is used to make the transverse shear strains completely consistent without introducing any spurious zero energy modes. Selectively reduced integration strategy and the assumed strain method are used to eliminate membrane locking (by making the membrane strain energy consistent) from the elements DSHL8 and SSHL8 respectively. Therefore, SSHL8 can be used to model the laminated composite structures more efficiently when compared to DSHL8. The parasitic shear is also eliminated from the formulations by making the in-plane shear strain component field-consistent. These elements are very accurate even when they are distorted to some extent.

SSHL9/DSHL9-9-noded degenerated shell elements: Single-surface and double-surface elements are also formulated using the biquadratic Lagrangian shape functions resulting in the corresponding 9-noded formulations - SSHL9 and DSHL9. Again the 3-dimensional elasticity is similarly degenerated to a 2-dimensional formulation. The geometric descriptions of the two versions are also similar to that of their serendipity counterparts.

These elements, in their conventional form, suffer from transverse shear locking (delayed convergence), membrane locking, parasitic shear and the associated stress oscillations. The transverse shear strain components are made field-consistent using the method of Legendre polynomial expansion without introducing any spurious zero energy modes. 
Selectively reduced integration strategy and the assumed strain method are used to eliminate the membrane locking (by making the membrane strain energy consistent) from the elements DSHL9 and SSHL9 respectively (not published). Again, SSHL9 is more efficient in modelling the laminated composite structures when compared to DSHL9. The parasitic shear is also eliminated from the formulations by making the in-plane shear strain component field-consistent. These elements are very accurate even when they are distorted to some extent.

\subsection{Elements for 3-dimensional elasticity}

A family of linear and quadratic hexahedral elements are developed based on an isoparametric formulation. The elements are based on 3-dimensional elasticity. They can take orthotropic and anisotropic material properties. These elements can be used to model any structure subjected to hygro-thermo-mechanical loads.

HEXA8 - A 8-noded hexahedral element: HEXA8 is an 8-noded brick element using the trilinear Lagrangian interpolation functions for element geometry as well as fielddescription. In its original form, it suffers from shear locking when used to model thin structures and near-incompressibility locking when used to model nearly incompressible structures and the associated disturbances in the stress recovery. It is made free of these errors by augmenting the displacement fields with the incompatible (bubble) functions and making the resultant constrained transverse shear and volumetric strains field-consistent (Chandra \& Prathap 1989).

HEXA20: A 20-noded hexahedral element: HEXA20 uses the 3-dimensional quadratic serendipity shape functions for interpolating the element geometry as well as the field variables. In its conventional form it suffers from both shear locking and near-incompressibility locking. These errors are alleviated using the consistency concepts in line with those used for the 8-noded plate elements in Prathap et al (1988). The resulting formulation is free of locking and stress oscillations, but suffer from spurious zero energy modes when used to model loosely constrained problems.

HEXA27: A 27-noded hexahedral element: HEXA27 is also an isoparametric formulation based on the triquadratic Lagrangian shape functions. In its original form, it suffers from locking and/or delayed convergence and stress oscillations. The line-consistency concepts are extended to 3-dimensional plane-consistency and the method of Legendre polynomial expansion is used to eliminate locking problems from this element (Naganarayana $\&$ Prathap 1991) without introducing any spurious zero energy mechanisms.

\section{The higher order transverse deformable elements}

The demand for accuracy in the transverse stress predictions - in thick and laminated structures - is increasing owing to the advanced structural applications, particularly in 
the aerospace and automobile industry. Such demands are being made frequently with the advent of high-precision design strategies, high computational capabilities and safety awareness. A full-fledged 3-dimensional solution for obtaining the transverse stress distribution is very expensive from a computational point of view. Recently, several higher-order transverse deformable plate/shell theories and the corresponding finite element formulations have been used for predicting more accurate transverse stress distributions over the plate thickness when compared to their elementary and first-order counterparts.

Higher order flexural deformation in plates has been modelled in many different fashions leading to existence of several higher order shear deformable theories (Lo et al 1977; Reddy 1984; Liao et al 1992). Out of the several theories, the Lo-Christensen-Wu theory is found to be the best candidate from the general finite element package point of view since it requires purely $C^{0}$-continuous field description.

\subsection{Theoretical basis}

The 3-dimensional displacement field is expanded in terms of the thickness coordinate explicitly such that the in-plane displacements are interpolated to a cubic level while the transverse displacement is interpolated to a quadratic level as:

$$
\begin{aligned}
& U(x, y, z)=u(x, y)+z \theta(x, y)+z^{2} u^{*}(x, y)+z^{3} \theta^{*}(x, y), \\
& V(x, y, z)=v(x, y)+z \phi(x, y)+z^{2} v^{*}(x, y)+z^{3} \phi^{*}(x, y), \\
& W(x, y, z)=w(x, y)+z \psi(x, y)+z^{2} w^{*}(x, y)
\end{aligned}
$$

thus reducing the 3-dimensional elasticity to a 2-dimensional problem. By substituting the above displacement field description into the regular 3-dimensional strain-displacement relations, we get a cubic description for the in-plane strain components, a parabolic description for the transverse shear strain components, and a linear description for the transverse normal strain component across the plate thickness.

\subsection{Finite element technology}

Again, from a general purpose applications point of view, finite element formulation should be free of all errors irrespective of their shape and thickness. In the limits of thin plates, the transverse shear and normal strain energy components are constrained to vanish in the higher order shear deformable element formulations. The specific consistency requirements for such elements and the variationally correct method of achieving the same have been recently evolved (Mohan et al 1994) so that the higher order shear deformable elements can model the above mentioned physical constraints correctly. It should be noted here that the transverse shear strains are made consistent in the thickness coordinate by suitably choosing the field description. The consistency requirements in the in-plane coordinates are explicitly achieved using the method of Legendre polynomial expansion. The elements are then made edge-consistent by matching the tangential transverse shear strain/stress components across the element boundaries in any arbitrary patch of elements and by mapping the constrained strain fields from the natural covariant space to the global Cartesian space in a consistent fashion (Naganarayana et al 1995). The elements based on 
such formulations perform very accurately, both in terms of displacements and stresses, even in their most distorted forms. A library of error-free beam (stiffener) (2-noded element BEAM2 and 3-noded element BEAM3) and plate elements (4-noded element QUAD4 and 9-noded element QUAD9) are developed based on consistency and correctness principles. Uniform full integration schemes are used in the element formulations. All the elements behave accurately in linear static, dynamic and instability applications. All elements can take laminated materials constitution with each lamina taking 3-dimensional orthotropic properties.

The complete library of laminated beam/plate elements that are currently available are being extended to tackle curvilinear laminated curved-beam/shell problems. This element library comprises the special module that is being planned to enhance FEPACS with extended interlaminar stress analysis capability as well as enhancing its current capability to tackle structures that are very thick and/or highly flexible in transverse deformation more accurately. With this complete module of higher order transverse deformable curvedbeam/shell elements, one can model any general stiffened laminated composite structures.

\section{Linear analysis capabilities}

The power of a general purpose finite element package is reflected by its capability to tackle a wide range of problems in its field of application. The solution capabilities, apart from the element technology that is used, mainly represent its versatility. Today, for structural applications, it is essential to have complete linear (static, dynamic and stability) solution capabilities in a general purpose finite element package.

Currently, FEPACS has the core solution capability of the original SAP software (linear static and dynamic (lumped mass) solution) which was recently enhanced to tackle distributed mass and linear stability as well. The solution can be performed in a single block (in-core solution) as well as in multiple blocks (out-of-core solution). Thus the solution capability can be utilised very efficiently for small as well as large problems as the requirement arises. Thus, the package can tackle any number of degrees of freedom provided the computer platform used can support the scratch file and dynamic memory requirements. The element matrices are assembled into their global counterparts in a banded form to optimise the memory requirement.

In this section, we shall briefly touch upon the salient features of the solution capabilities of FEPACS and the recent developments that are underway to improve/replace the present module.

\subsection{Linear static analysis}

A typical static structural analysis involves solving a set of simultaneous equations representing the structural equilibrium:

$$
[K] u=f,
$$

where $[K]$ is the structural stiffness, $f$ is the vector of applied nodal forces, and $u$ is the vector of the unknown nodal degrees of freedom. A Gauss elimination algorithm is used to decompose the positive definite symmetrical system of equations. The algorithm 
is optimised to a certain extent: no operations on the zero elements, load vectors are reduced at the same time as the stiffness matrix is decomposed.

Once the equilibrium equations are solved for the nodal displacements, the element stresses are computed from the element nodal displacements extracted from the displacement solution using the same strain-displacement and constitutive relations that were used in computing the element stiffness. Stresses are recovered accurately all over the element domain since the element technology used is based on the consistency and correctness principles. Stresses can, normally, be recovered at either element nodes or at the popular Barlow points for each element. The stress/strain fields are reconstituted in accordance with the unconstrained-field consistency paradigm such that the results are variationally correct even when the sectional properties of the structure varies over an element.

\subsection{Linear natural frequency analysis}

Free vibration analysis is one of the basic requirements in structural design in many fields of applications - aerospace, offshore, seismic, automobile etc. Typically, the problem is to solve a set of homogeneous equations,

$$
[K] \phi=\omega^{2}[M] \phi,
$$

where $[M]$ is the structural mass matrix; and $\omega$ and $\phi$ are the natural frequency and mode shape respectively. The above eigenvalue problem is solved for the lowest natural frequencies using two distinct solution procedures: determinant search method and subspace iteration technique (Bathe \& Wilson 1973). The determinant search solution is carried out when the element matrices can be contained in the high-speed or active memory in one block. On the other hand, for systems of large order and bandwidth the subspace iteration method is used such that the equilibrium equations are tackled in multiple blocks. Both solution techniques solve the generalised eigenvalue problem directly without a transformation to the standard form (Bathe \& Wilson 1973). Originally, only lumped mass was considered. Recently, the option of distributed mass was also included (Naganarayana et al 1993) into FEPACS so that one can obtain a better model for the free structural vibrations, since the element library is basically based on a shear deformable theory and hence can depict secondary frequency spectra accurately (Bhashyam \& Prathap 1981).

\subsection{Linear dynamic analysis}

For the response history analysis of structures subjected to dynamic loads we seek solution to the dynamic equilibrium equations,

$$
[M] \mathrm{d}^{2} u / \mathrm{d} t^{2}+[C] \mathrm{d} u / \mathrm{d} t+[K] u=f,
$$

where $M, C, K$ are the mass, damping and stiffness matrices of the structure, and $f$ and $u$ are the transient nodal force and displacement vectors. The damping matrix is computed as a linear combination of the mass and stiffness matrices such that the modal orthogonality is satisfied. As a variation, the dynamic equilibrium equations can also handle structures subjected to uniform ground acceleration by replacing the right hand side by $-[M] \mathrm{d}^{2} u_{g} / \mathrm{d} t^{2}$ where $u_{g}$ is the ground displacement and $u$ is the structural displacement 
with reference to the ground. The dynamic structural response analysis problem, can be carried out in two ways using FEPACS: natural frequency analysis followed by response history analysis using the mode superposition method, and response history analysis by direct integration (Clough 1962).

In another variation of dynamic analysis, with a particular reference to seismic problems, the Cartesian components of the ground acceleration can be used to carry out the response spectrum analysis using the concept of spectral displacement (Clough 1962). FEPACS computes the maximum responses for each mode where the spectra (displacements or accelerations) in the three directions are assumed to be proportional to each other.

\subsection{Linear stability analysis}

The linear buckling analysis of a structural system is carried out as an eigenvalue solution,

$$
[K] \phi=\lambda[G] \phi,
$$

where $G$ is the structural geometric stiffness; $\lambda$ represents the critical load factor; and $\phi$ is the corresponding buckling mode. It is assumed that the pre-buckling structural behaviour is linear in nature and that the internal force distribution is linearly proportional to the applied reference force. Then, the applied reference load multiplied by the critical load factor $\lambda_{c r}$ represents the buckling load configuration.

Sometimes, the internal force distribution is known a priori for a given load configuration. In such cases, the elastic and geometric stiffness matrices are computed for the system and the eigenvalue solution is carried out directly (single phase strategy) to find the buckling loads and modes. However, very often, the internal force distribution has to be computed explicitly for a problem with certain specified topology, boundary conditions and material constitution. In such cases a multi-phase strategy is adapted wherein a preliminary static analysis is carried out to find the internal force distribution induced in the structure by the reference loads which is then used to find the structural geometric stiffness. The elastic stiffness computed in the first phase and the geometric stiffness computed in the second phase are then fed for the final phase of eigenvalue solution to obtain the critical buckling loads and the associated modeshapes. Thus, FEPACS can be used to analyse simple well-defined as well as general structural instability problems very efficiently and accurately (Naganarayana et al 1993).

\subsection{Frontal solution module}

The method of solving the equilibrium equations is a major factor influencing the computational time and memory requirement in general finite element structural analysis. This becomes very important, particularly in case of nonlinear and/or dynamic finite element analyses, where several equilibrium iterations have to be performed for several load/time/displacement increments. From the point of computational efficiency, time and memory, frontal solution techniques are becoming more and more popular recently since these techniques can possibly give an edge over the conventional active-column solution strategy as the one existing in the current version of FEPACS.

In frontal solution technique (Irons 1970), the complete assembly of the global matrix is never carried out explicitly. The process of assembly of elements and elimination of 
variables are carried out simultaneously. Typically, the degrees of freedom associated with a structural node are statically condensed out of the solution system as soon their interaction with the nodes on the boundary surrounding the node is well established in the simultaneous process of continuing assembly of element matrices and the equilibrium solution. Thus the distinctive feature of frontal method is storing only the active equations in the core memory. This feature leads to low core memory requirement. Apart from this, the frontal method has several other advantages as well. Back-substitution for different load vectors requires very little core memory. This feature is particularly attractive in the context of nonlinear analysis or eigen solution where many resolution with different righthand-side vectors are required. It is much easier to add static condensation and, therefore, the substructuring capabilities into the solution capabilities. Since the assembly and the solution processes are reasonably parallel in nature, frontal solution is more convenient for parallelising and/or vectorisation.

A complete solution module for linear static, instability and dynamic structural analysis is being planned in a stand-alone mode. This module could be tailored to be absorbed in FEPACS subsequently. It is also planned to use the frontal solution capabilities for the forthcoming nonlinear finite element modules, that are being developed, independently. It is currently aimed at including some special modelling facilities to handle multiple load cases, fixed or rigid boundary conditions, prescribed displacement boundary conditions, multipoint constraints, skew boundary conditions, lumped and/or consistent mass matrix, concentrated mass at arbitrary nodes, static condensation and substructuring, and various types of elements that are in the current FEPACS version.

In the first phase of the work, the basic frontal algorithm has been developed for static solution and all the modelling features mentioned above have been incorporated. In the next phase, it is planned to include a general eigenvalue solution capability such that linear buckling and free-vibration analysis can be performed. In the third phase, forced response computation capabilities would be included to complete the solution capabilities in-par with the current regular solution features of FEPACS.

\section{Composites applications}

Laminated composite structures are gaining importance in many applications, particularly in the fields of aerospace, automobile and naval engineering. Their growing popularity can be traced to many desirable properties they possess, such as, very high weight-tostrength and weight-to-toughness ratios, excellent fatigue resistance, tailorable directional thermo-mechanical properties, reduced part count over their metallic equivalents etc. They have been routinely used to produce many structural components as well. Today, therefore, the thrust of general purpose computational structural mechanics is to enable one to model general laminated composite structures subjected to hygro-thermo-mechanical loads.

In FEPACS, bending elements are allowed to have a general laminated construction such that each lamina can take isotropic, orthotropic or anisotropic material properties with distinct thicknesses. The formulation for modelling laminated structures with 3-dimensionally orthotropic laminae is presented below. The model can take any number of layers with 
any arbitrary fibre orientation and thickness. The mechanical strains experienced by the structure are given by,

$$
\epsilon_{m}=\left(\begin{array}{r}
(\epsilon+z \kappa)-\left(\epsilon_{o}+z \kappa_{o}\right) \\
\gamma
\end{array}\right),
$$

where $\epsilon, \kappa$ and $\gamma$ are the total membrane, bending and transverse shear strains, and $\epsilon_{0}$ and $\kappa_{0}$ are the initial (thermal) membrane and bending strains proportional to midsurface temperature $T$ and temperature gradient $\Delta T$ respectively. The constitutive relation for the anisotropic medium is given by,

$$
\boldsymbol{\sigma}=\mathbf{Q} \epsilon_{m}
$$

where $\mathbf{Q}$ is the constitutive matrix containing 21 independent constants. Normally, laminated structures are composed of laminae with 3-D orthotropic properties. Depending on the type of lamina materials, $\mathbf{Q}$ can be appropriately computed. If $P$ is the potential of the applied loads, the total potential energy for the system is given by

$$
\mathbf{P}_{M T P E}=\frac{1}{2} \int_{v} \boldsymbol{\epsilon}_{m}^{T} \mathbf{Q} \epsilon_{m} d v-P .
$$

Substituting (6) and (7) in (8), and applying the minimum total potential energy principle, we get the structural equilibrium equations as,

$$
\begin{aligned}
& \int_{A}\left(\delta \epsilon^{T}(\mathbf{A} \epsilon+\mathbf{B} \kappa-\mathbf{F} T-\mathbf{G} \Delta T)\right. \\
& \left.\quad+\delta \kappa^{T}(\mathbf{B} \boldsymbol{\epsilon}+\mathbf{D} \boldsymbol{\kappa}-\mathbf{G} T-\mathbf{H} \Delta T)+\delta \gamma^{T} \mathbf{E} \gamma\right) d A=\delta P
\end{aligned}
$$

where, if $\hat{\mathbf{Q}}$ and $\overline{\mathbf{Q}}$ represent the constitutive matrices correlating the inplane and transverse deformation respectively,

$$
\begin{aligned}
(\mathbf{A} B \text { B } D) & =\sum_{l=1}^{L} \int_{z_{l}} z_{l+1} \hat{\mathbf{Q}}_{l}\left(1 \quad z \quad z^{2}\right) \mathrm{d} z \\
(\mathbf{F ~ G ~} B H) & =\sum_{l=1}^{L} \int_{z_{l}} z_{l+1} \hat{\mathbf{Q}}_{l} \alpha\left(1 z z^{2}\right) \mathrm{d} z \\
\mathbf{E} & =\sum_{l=1}^{L} \int_{z_{l}} z_{l+1} \overline{\mathbf{Q}}_{l} \mathrm{~d} z
\end{aligned}
$$

and $\boldsymbol{\alpha}$ is the vector of thermal expansion coefficients.

In case of anisotropic construction, the structural constitutive relations, matrices $\mathbf{A}, \mathbf{B}$, D, E, F, $\mathbf{G}$ and $\mathbf{H}$, have to be explicitly supplied to FEPACS. This 2-dimensional model is used in formulating the plate/shell elements - SHEL4, SSHL8, DSHL8, SSHL9 and DSHL9. For the one-dimensional elements - EBEAM2, TBEAM2 and TBEAM3 - these 2-dimensional relations are statically condensed to get the equivalent one-dimensional constitutive relation.

The higher order shear deformable laminated composite beam/plate elements $(\S 5)$ are also formulated in a similar way. These elements can be effectively used for refined linear interlaminar stress analysis and for linear dynamic and instability analysis of laminated plates and beams under hygro-thermal and mechanical loads (Mohan et al 1994). 


\section{Hygro-thermal loads}

Recently, it was observed that displacement type elements suffer from extraneous stress oscillations when used to model hygro-thermal loads (Prathap \& Naganarayana 1994). It was shown that it was due to violation of the unconstrained-field consistency paradigm. Solutions are offered to eliminate these errors in first order shear deformable and 1-, 2and 3-D elasticity formulations by reconstituting the initial strain fields to achieve consistency with reference to the corresponding total strain fields. The orthogonality conditions required are derived by taking an equivalence of the single-field minimum total energy principle with the general three-field $\mathrm{Hu}$-Washizu principle.

The consistent, correct and complete analysis capability is implemented in FEPACS for hyggro-thermal stress analysis of general laminated composite structures. FEPACS considers a temperature description that is compatible with the displacement field description. For example, both mid-surface temperature and temperature gradient across the element thickness are considered in case of bending elements and the coupled membrane-flexural structural behaviour is captured. This becomes particularly important in applications to laminated composite structure where the in-plane and flexural deformations can be implicitly coupled.

The consistency paradigms - related to the initial strain problems - are recently extended to the higher order shear deformable element formulations. It is noticed that the inconsistent hygro-thermal strain field description can also affect the constrained-field consistency requirements apart from the above mentioned unconstrained-field consistency requirements of an element. The consistency paradigms are judiciously applied leading to optimal element formulations. All the elements in the library of higher order shear deformable elements are now reconstituted to tackle the hygro-thermal problems in laminated composite structures in a consistent and correct manner. Apart from consistent hygro-thermo-mechanical stress analysis, it is now possible to compute the combined influence of the moisture content, the temperature distribution and the mechanical loads on structural vibration and buckling as well. This capability is being consolidated with the module of the higher order shear deformable element library.

\section{Nonlinear finite element analysis}

Today, nonlinear structural analysis is becoming more and more important, acknowledging the increasing demand for high precision applications, use of advanced materials, and for safe design procedures, particularly in aerospace and automobile applications, apart from conventional fields such as metal forming and many other manufacturing techniques. A structure can experience mainly two types of nonlinearities - geometric (where kinematic relations are nonlinear) and material (where constitutive relations are nonlinear) - apart from other phenomena like contact, friction etc. Very often nonlinear structural behaviour is coupled with (macro-) structural instabilities (e.g. buckling) and/or material or microstructural instabilities (e.g. necking, shear band formation etc.). As a first step toward having a complete nonlinear capability in FEPACS, work has been in progress in developing fully automated geometrically nonlinear analysis capability for structures with possible structural instabilities. 
Element technology: Efforts have been initiated toward developing an error-free finite element technology for geometrically nonlinear applications (Naganarayana \& Prathap 1996). The causes for the nonlinear locking and the associated disturbances in the stresses have been located and eliminated in a variationally correct manner in formulating robust curved beam elements. We plan to extend the technology to the 2- and 3-dimensional elements as well to complete the element library from the geometric nonlinearity point of view.

Automated solution strategies: Recently, the different steps involved in a typical automated nonlinear and/or post-buckling solution strategy are identified; different strategies that are evolved for each step are compiled and examined critically for their comparative performance in optimal combinations of strategies for best performance in geometrically nonlinear applications with different kinds of possible instabilities such as limit points and bifurcation points (Naganarayana 1995). Such strategies are currently being implemented in a modular fashion for general finite element applications.

\section{Pre- and post-processing}

Keeping in mind the vast usage of the finite element method for real-state problems, it has become nearly impossible to depend on manual data preparation and result interpretation. A pre-/post-processor has, therefore, become an integral part of finite element analysis. Efforts are being made to enhance FEPACS utility from the real general purpose applications point of view with a Graphical User Interface (GUI).

Recently, FEPACS was interfaced with a commercially available pre-/post-processing software released by MSC - MSC-XI - which intrinsically supports the MSC/NASTRAN data structure (Geetha \& Naganarayana 1995). The sort and search algorithms of NASTRAN are emulated in the interface software, FEPNAST, to interpret NASTRAN data in FEPACS format. The complete element library of FEPACS is not directly supported by MSC-XL. Data related to the unsupported elements are indirectly emulated, e.g. the quadratic elements of FEPACS are generated using an appropriate MSC-XL model generated using the linear elements of NASTRAN.

Currently, another commercial pre- and post-processing software released by EMRC DISPLAY-III - which supports the finite element package, NISA, is being interfaced with FEPACS. The data handling structure of NISA is reproduced to interpret the NISA data deck generated by DISPLAY-III and the data is written to a file conforming to the FEPACS format. The interface module, FEPNIS, is currently capable of interfacing the basic linear elements of the NISA element library for static analysis with FEPACS.

Finally, efforts are being made to develop an indigenous GUI that can be used for preand post-processing for general purpose finite element modelling and analysis to support FEPACS. It is planned to develop the software in C-language under the UNIX operating system. The X-developmental tools and Motif are being used for the menu/window operations and the graphics. The skeletal infrastructure is developed for incorporating the slots for desirable capabilities of a pre- and post-processor to be filled in due course of time - NALGRAF. As an initial step, a post-processing module is implemented in the 
infrastructure for presentation graphics. Currently, a general purpose geometric modelling module with manual support as well as with an expert advisor support is being developed for incorporation in the infrastructure.

\section{Structural damage assessment/prediction}

Damage is one of the most important criteria for safe design, particularly in aerospace and automobile applications. And, composites are becoming the preferred candidates for many structural applications due to their obvious superiority over their metal counterparts, particularly from the specific strength point of view. Their applications are still limited since their behaviour is not understood properly from a damage mechanics point of view. Accordingly, failure mechanics in composite structures has been one of the thrust areas of research in the literature for the past 2 to 3 decades.

Recently, work has been initiated to develop a damage module for FEPACS. As a first step, failure mechanisms in delaminated structures have been critically examined taking the geometric nonlinearity of the structure and delamination propagation into consideration. A simple method is evolved to model the delaminated stiffened composite structures, to predict delamination growth in terms of pointwise energy release rate distribution along the delamination edge, to trace the multiple post-buckling deformation modes, and to predict the residual strength of the damaged structure (Naganarayana \& Atluri 1996).

\section{Expert advisor for finite element analysis}

It is very desirable to carry out tasks that involve high levels of expertise, experience and judgement even in the absence of the required experts. Today, this desire has come close to fulfilment to some extent due to the advances in software technology. Expert system activities are initiated (Naganarayana \& Prathap 1992) keeping enhancement of FEPACS capabilities in mind. An expert advisor was first incorporated into a 3-dimensional finite element package for problem modelling and adaptive mesh control (Prathap \& Naganarayana 1992). Later, an expert advisor was devised for the general purpose finite element modelling of the 2-dimensional structures using the C-language in the DOS environment (Naganarayana et al 1994). It is now planned to develop a full-fledged expert system for general purpose finite element modelling as a module in NALGRAF - the pre-/post-processor that is being developed at NAL (see $\S 10$ ). Currently, as a first step toward this goal, the solid modelling and graphics capabilities are being enhanced with reference to the experience and expertise acquired over the past few years. The software is developed in $\mathrm{C}$. The menu operations and graphics are supported by the $\mathrm{X}$-development tools and Motif in the UNIX operating system environment.

\section{Finite element structural optimisation}

A structural optimisation module is being developed for integration with FEPACS. The module is being planned to incorporate several state-of-the-art Mathematical Programming Techniques available in the literature for structural optimisation (Haftka \& Kamat 1985). 
In the first phase of development, constraints based on static consideration only will be considered with the objective being weight minimisation; the basic structural elements TRUSS, BEAM2 and SHEL4 - will be supported; and a design sensitivity analysis based on both displacements and stresses will be incorporated.

Using FEA alone for repeated analysis during the process of optimisation is quite expensive. Hence, approximate methods (Schmit \& Miura 1976) are devised (e.g. Taylor's series expansion to define the approximate analysis problem) such that the constraints and the objective function values can be quickly evaluated with reasonable accuracy and much less expense. Currently, each FEA cycle is followed by several approximate analysis cycles and the sequence is repeated until the results converge.

\section{Conclusions}

In this paper, we intended to outline the research and development activities involved in developing the general purpose Finite Element Package for Analysis of Composite Structures (FEPACS). The element technology, modelling and solution capabilities of FEPACS are briefly described. The current version of FEPACS has the state-of-the-art element technology based on what are called the $\mathrm{C}$-concepts. It incorporates a complete library of finite elements (1-, 2- and 3-dimensional linear and quadratic). Each element is free of all errors of locking, delayed convergence and stress oscillation even in its most general form. The package, currently, has the linear static, instability and dynamic (free/forced vibrations) analysis capabilities. It can model any general isotropic, orthotropic, anisotropic, and laminated composite structure subjected to hygro-thermo-mechanical loads.

Currently, several research and development activities are in progress in an effort to enhance the FEPACS capabilities both in terms of utility and applicability to a wider range of problems. The current linear solution capability of FEPACS is being augmented by an independent module based on frontal strategies. Work is in progress to evolve automated general nonlinear solution capability for finite element applications. From pre- and postprocessing point of view, FEPACS is now being supported by two commercially available GUI's - MSC-XL on UNIX platform and DISPLAY-III on DOS platform. An indigenous GUI is being developed in the C-language using X-developmental tools and Motif kit for graphical support on UNIX platform. An expert advisor for structural modelling, finite element modelling and structural analysis is being developed in association with such a GUI. A module for structural optimisation is also under progress to be incorporated with FEPACS. Finally a module for damage assessment and/or prediction is also initiated particularly for composite applications. In this paper, all these activities were also briefly touched upon.

The authors are grateful to Prof. R Narasimha and Dr. K N Raju for their constant encouragement and keen interest in the activities. They also acknowledge Dr. K Guruprasad, M/s S Nagaraj anc B Sudhakar for helping in installing the software on different platforms; Mr Bhaskar, Mr Cheman, Ms Shashirekha, Ms Geetha, Ms Manju and Dr. Rajashekar for their support in validation of the software and in documentation. 


\section{References}

Ahmed S, Irons B M, Zienkiewicz O C 1970 Analysis of thick and thin shell structures by curved finite elements. Int. J. Numer. Methods Eng. 2: 419-451

Barlow J 1976 Optimal stress locations in finite element models. Int. J. Numer. Methods Eng. 10: 243-251

Bathe K J, Wilson E L 1973 Solution methods for eigenvalue problems in structural mechanics. Int. J. Numer. Methods Eng. 6(2)

Bathe K J, Wilson E L, Peterson F E 1974 SAP IV - A structural analysis program for static and dynamic response of linear systems. College of Engineering, University of California, Berkeley

Bhashyam G R, Prathap G 1981 Second frequency spectrum of Timoshenko beams - a reassessment. J. Sound Vib. 76: 407-420

Chandra S, Prathap G 1989 A field-consistent formulation for the 8-noded solid finite element. Comput. Struct. 33: 345-355

Clough R W 1962 Earthquake analysis by response spectrum superposition. Bull. Seismol. Soc. Am. 52:

Cook R D 1975 Avoidance of parasitic shear in plane element. J. Struct. Div., Am. Soc. Civil Eng. 93: 43-66

Cook R D, Malkus D S, Plesha M E 1981 Concepts and applications of finite element analysis 3rd edn (New York: John Wiley and Sons)

Doherty W'P, Wilson E L, Taylor R L 1969 Stress analysis of axisymmetric solids using higher order quadrilateral elements. SESM Report 69-3, Dept. Civil Eng., University of California, Berkeley

Fried I 1974 Finite element analysis of incompressible material by residual energy balancing. Int. J. Solids Struct. 10: 993-1002

Geetha B, Naganarayana B P 1995 FEPNAST - An interface between MSC/XL and FEPACS. PD ST 9509, National Aerospace Laboratories, Bangalore

Haftka R T, Kamat M P 1985 Elements of structural optimisation (Martinus Nijhoff)

Hughes T J R 1987 The finite element method: Linear static and dynamic finite element analysis (Englewood Cliffs, NJ: Prentice Hall)

Irons B M 1970 A frontal solution program. Int. J. Numer. Methods Eng. 2: 5-32

Lo K H, Christensen R M, Wu E M 1977 A higher order theory of plate deformation. Part-1: Homogeneous plates; Part-2: Laminated plates. ASME J. Appl. Mech. 44: 663-668, 669-676

Liao M L, Jing H S, Hwang M 1992 Improvements on the higher order plate element with partial hybrid stress model. Comput. Struct. 42: 45-51

Mackerle J 1995 Some remarks on progress with finite elements. Comput. Struct. 55: 1101-1106

MacNeal R H 1982 Derivation of element stiffness matrices by assumed strain distributions. Nucl. Eng. Design 70: 3-12

MacNeal R H 1994 Finite elements: Their design and performance (New York: Marcel Dekker) Mohan PR, Naganarayana B P, Prathap G 1994 Consistent and variationally correct finite elements for higher order laminated plate theory. Compos. Struct. 29: 445-456

Naganarayana B P 1991 Consistency and correctness principles in quadratic displacement type finite elements $\mathrm{Ph} \mathrm{D}$ dissertation, Indian Institute of Science, Bangalore

Naganarayana B P 1995 Incremental iterative strategies for automated post-buckling analysis. Comput. Struct. (in press)

Naganarayana B P, Atluri S N 1996 Computational modelling and analysis of coupled failure in delaminated composite structures. Sādhanā 21: 547-575 
Naganarayana B P, Prathap G 1989a Force and moment corrections for the warped four-node plane shell element. Comput. Struct. 33: 1107-1115

Naganarayana B P, Prathap G 1989b Displacement and stress predictions from field- and lineconsistent versions of the 8-noded Mindlin plate element. Comput. Struct. 33: 1095-1106

Naganarayana B P, Prathap G 1990 Consistency aspects of out-of-plane bending, torsion, and shear in a quadratic curved beam element. Int. J. Numer. Methods Eng. 30: 431-443

Naganarayana B P, Prathap G 1991 Field-consistency analysis of 27-noded hexahedral elements for constrained media elasticity. Finite Elements Anal. Design 9: 149-168

Naganarayana B P, Prathap G 1992 Expert systems and finite element structural analysis - a review. Sädhanā 17: 275-298

Naganarayana B P, Prathap G 1996 Locking in geometrically nonlinear beam elements. AIAA J. (Communicated)

Naganarayana B P, Shashirekha B R, Prathap G 1993 Implementation of linear buckling capabilities into FEPACS. PD ST 9318, National Aerospace Laboratories, Bangalore

Naganarayana B P, Virupaksha S A, Prathap G 1994 Design and development of an expert system advisor for general purpose modelling of 2-dimensional structures. PD ST 9401, National Aerospace Laboratories, Bangalore

Naganarayana B P, Mohan P R, Prathap G 1995 Quadrilateral $C^{0}$ laminated plate elements based on a higher order transverse deformation theory. Int. J. Eng. Anal. Design 2: 157-178

Pawsey S E, Clough R W 1971 Improved numerical integration of thick shell finite elements. Int. J. Numer. Methods Eng. 3: 454-586

Pian T H H 1971 Hybrid models Numer. Comput. Methods Appl. Mech. (special issue)

Prathap G 1985 The poor bending response of the 4-noded plane stress quadrilateral element. Int. J. Numer. Methods Eng. 21: 825-835

Prathap G 1986 Field-consistency - Toward a science of constrained multi-strain-field finite element formulations. Sädhanā 9: 319-344

Prathap G 1988 The variational basis for least squares field-redistribution of strain functions in the finite element formulations of constrained media elasticity. TM ST 8801, National Aerospace Laboratories, Bangalore

Prathap G 1993 Finite element method in structural mechanics (Dordrecht: Kluwer)

Prathap G 1994 Finite element method in structural mechanics. Prog. Aerosp. Sci. 30: 295-405

Prathap G 1996 The stress correspondence paradigm in finite element analysis. Sädhanā 21: (this issue)

Prathap G, Bhashyam G R 1982 Reduced integration and the shear flexible beam element. Int. J. Numer. Methods Eng. 18: 195-210

Prathap G, Naganarayana B P 1990a Consistent force resultant distributions in displacement elements with varying sectional properties. Int. J. Numer. Methods Eng. 29: 775-783

Prathap G, Naganarayana B P 1990b Analysis of locking and stress oscillations in a general curved beam element. Int. J. Numer. Methods Eng. 30: 177-200

Prathap G, Naganarayana B P 1991 FEPACS, A finite element package for analysis of composite structures. PD ST 9139, National Aerospace Laboratories, Bangalore

Prathap G, Naganarayana B P 1992a Field-consistency rules for 3-noded shear flexible beam elements under nonuniform isoparametric mapping. Int. J. Numer. Methods Eng. 33: 649-664

Prathap G, Naganarayana B P 1992b A 3-D finite element package with expert system and adaptive mesh control: Concluding report. PD ST 9102, National Aerospace Laboratories, Bangalore

Prathap G, Naganarayana B P 1994 Consistent thermal stress evaluation in finite elements. Comput. Struct. 54: 415-426

Prathap G, Somashekar B R 1988 Field-and edge-consistency synthesis of a 4-node quadrilateral 
plate bending element. Int. J. Numer. Methods Eng. 33: 649-664

Prathap G, Naganarayana B P, Somashekar B R 1988 Field-consistency analysis of the isoparametric 8-node plate bending element. Comput. Struct. 29: 857-873

Prathap G, Dattaguru B, Naganarayana B P 1989 Development of general purpose finite element package for structural analysis of isotropic, anisotropic and laminated composite structures. Project Proposal to AR and DB, PP ST 8907, National Aerospace Laboratories, Bangalore

Prathap G, Naganarayana B P, Somashekar B R 1994a Development of robust finite elements for general purpose structural analysis. Sādhanã 19: 289-309

Prathap G, Naganarayana B P, Somashekar B R 1994b FEPACS (Version 2.0): Finite element package for analysis of composite structures - Theoretical manual. PD ST 9405, National Aerospace Laboratories, Bangalore

Ramesh Babu C 1985 Field-consistency in the finite element formulation of multi-strain-field problems in structural mechanics. $\mathrm{Ph} \mathrm{D}$ dissertation, Indian Institute of Technology, Madras

Ramesh Babu C, Somashekar B R, Prathap G 1985 Development of a library of field-consistent elements. J. Aeronaut. Soc. India 37: 327-335

Reddy J N 1984 A simple higher order theory for laminated composite plates. J. Appl. Mech. 51: $745-752$

Schmit L A Jr, Miura H 1976 Approximate concepts for efficient structural synthesis. NASA CR 2552

Stolarski H, Belytchko T 1981 Membrane locking and reduced integration for curved elements. J. Appl. Mech. 49: 172-178

Tang L, Chen W, Liu Y 1984Formulation of quasi-conforming element and $\mathrm{Hu}-W a s h i z u$ principle. Comput. Struct. 19: 247-250

Wilson E L 1970 SAP - A general structural analysis program. SESM Report 70-20, Dept. of Civil Engineering, University of California, Berkeley.

Wilson E L, Taylor R L, Doherty W P, Ghabussi T 1973 Incompatible displacement models. Numerical and computational methods in structural mechanics (eds) ST Ferves et al (New York: Academic Press) pp 43-66

Zienkiewicz O C 1977 The finite element method 3rd edn (London: McGraw Hill)

Zienkiewicz O C, Taylor R L, Too J M 1971 Reduced integration techniques in general analysis of plates and shells. Int. J. Numer. Methods Eng. 3: 275-290 